\title{
Mobile Sensor Networks Based on Several Algorithms
}

\author{
Zhang Li \\ Department of Mathematics \& Physics Hohai University, Changzhou, China \\ Emails: zhangl@hhuc.edu.cn
}

Keywords: flocking, localization, dispatch, aggregation, mobile sensor networks.

Abstract: In this paper, we propose several algorithm for mobile sensor networks. Such that distributed flocking algorithm, node dispatch algorithm, node localization algorithm, aggregation algorithm etc.The distributed flocking algorithm can attain a more uniform deployment of sensors. the node dispatch algorithm can effectively reduce the amount of message transmission and the whole algorithm can efficiently extend system lifetime. node localization algorithm can improve the positioning accuracy and convergence rate, and can well apply to the environment of low anchor node density. the aggregation formed by algorithm is appropriate scale and the time cost is moderate growth.

\section{Introduction}

Sensor networks have been widely used in various applications, such as military sensing, environment monitoring, traffic surveillance [1]. In some scenarios, sensors can be manually placed over the area of interest (AOI) or around a target of interest (TOI) to ensure the successful completion of tasks. However, the manual deployment of sensors is impracticable due to its high cost of manpower and, furthermore, in certain hazardous environments such as disaster regions and battlefields. In view of this point, the random placement attracts more attention lately. However, it is difficult to guarantee that the random placement of sensors can achieve the desired performance goals of the network. In this situation, sensors have to be movable and their locations can be self-adjusted. This is also important to the environment of applications changes over time.Optimal sensor deployment is a challenging problem which attracts much attention lately. Maximal coverage of the AOI is an important objective to guide the sensor deployment. For example, if the sensor range is described as a disk centered at a sensor, the ratio of the covered area to the whole area of the AOI can be used a metric to evaluate the quality of a deployment algorithm [2]. The worst-case coverage is also adopted in the sensor deployment [3]. It measures the probability that a target travels across an AOI without being detected. To this end, a practical target detection probability has to be taken into account. In [4] and [5], researchers consider the sensor deployment which aims to employ the least number of sensors to meet the coverage requirement. For a mobile sensor network, the sensor deployment problem can be formulated as a combinatorial optimization problem, which maximizes the detection probability under the deployment constraints [6]. The generic algorithm is employed to tackle the resulting problem. An incremental strategy is presented in [7], where sensors are placed one by one into an AOI based on the information of sensors deployed earlier. Three Voronoi diagrams based algorithms are proposed in [8] to detect and fix coverage holes. The first algorithm (namely, VEC) maximize the coverage area by pushing away sensors from each other, while the second one (namely, VOR) pulls sensors toward the holes. In the third algorithm, Minimax, the coverage holes are filled in by relocating a sensor to the center of the smallest enclosing circle of its Voronoi vertices, such that the sensor collision can be avoided. In [9], 
an adaptive triangular deployment algorithm is developed to maximize the coverage area while minimizing the coverage overlaps. This approach adjusts the relative distance of each sensor to its neighbors to maintain a equilateral triangle configuration, that is proven to be the best hole-free structure with maximal coverage.

\section{flocking-based mobile sensor deployment}

This text assume that a mobile sensor network consists of $\mathrm{N}$ sensors in two- or three-dimensional space. Each sensor's position is denoted by $x_{i}$ for $i=1,2, \mathrm{~K}, N$. The network is modeled by a graph $\mathrm{g}=(v, \varepsilon)$ wherevrepresentsthe set of sensors. Let the neighbor set of a sensor be $\mathbf{N}_{i}=\left\{j \mid j \in \mathbf{V}, j \neq i,\left\|\mathbf{x}_{i}-\mathbf{x}_{j}\right\|_{2} \leq R\right\}$, where $\|\cdot\|_{2}$ denotes the Euclidean norm and $\mathrm{R}$ is the communication range of a sensor. Then, eis the set of edges linking each pair of sensors in neighborhood, i.e., $\mathbf{E}=\left\{(i, j) \mid i, j \in \mathrm{V}, j \in \mathbf{N}_{i}\right\}$. The adjacency matrix $\mathrm{A}=\left[a_{i j}\right]$ of $\mathrm{G}$ is a matrix where $a_{i j} \in[0,1], a_{i i}=0$, and $a_{i j} \neq 0$ if and only $(i, j) \in \mathbf{E}$. Inthis paper, we adopt $\left\{a_{i j}\right\}$ defined in [10]

$$
\begin{gathered}
a_{i j}=\rho_{h}\left(\left\|\mathbf{x}_{i}-\mathbf{x}_{j}\right\|_{\sigma} /\|R\|_{\sigma}\right) \\
\text { Where } \quad \rho_{h}(z)= \begin{cases}1, & 0 \leq z<h \\
\frac{1}{2}\left[1+\cos \left(\pi \frac{z-h}{1-h}\right)\right], & h \leq z \leq 1 \\
0, & \text { otherwise }\end{cases} \\
\|z\|_{\sigma}=\frac{1}{\varepsilon}\left[\sqrt{1+\varepsilon \|_{2}^{2}}-1\right] .
\end{gathered}
$$

The gradient of $\|z\|_{\sigma}$ is given by $\sigma_{\varepsilon}(z)=\frac{z}{\sqrt{1+\varepsilon\|z\|_{2}^{2}}}$.

Suppose that a TOI is located on $\mathbf{x}_{T}$. The objective of mobile sensor deployment under consideration is to uniformly place these sensors around the TOI. It has been shown [9] that three neighboring sensors should constitute an equilateral triangle such that their coverage area is maximized and the overlap area is minimized. In practice, if sensors are manually placed, this requirement can be easily satisfied. But in some scenarios, sensors can only be initially deployed at random. Then, they automatically move to the positions which satisfythe requirement aforementioned. We assume that each sensor moves with second-order dynamics

$$
\begin{cases}\dot{x}_{i} & =\mathbf{v}_{i} \\ \mathbf{v}_{i} & =\mathbf{u}_{i}\end{cases}
$$

where $\mathrm{u}_{i}$ represents a stimulus which determines the acceleration. Let $\mathrm{U}(\mathrm{x})$ be the collective potential function, which is differentiable and nonnegative. The global minimum of $U(x)$ should 
correspond to the sensor configuration where neighboring sensors are equally distant to each other, i.e., $\left\|\mathbf{x}_{i}-\mathbf{x}_{j}\right\|_{2}=d \leq R$ for $\forall i \in \mathrm{V}$ and $\forall j \in \mathrm{N}_{i}$. The value of $\mathrm{d}$ could be determined based on practical communication model. To this end, the pairwise potential function is further defined

$U_{i j}(\mathbf{x})=\Phi\left(\left\|\mathbf{x}_{j}-\mathbf{x}_{i}\right\|_{\sigma}\right)$

where

$$
\begin{gathered}
\Phi(z)=\int_{\|d\|_{\sigma}}^{z} \varphi(s) d s \\
\varphi(z)=\rho_{h}\left(z /\|R\|_{\sigma}\right) \omega\left(z-\|d\|_{\sigma}\right)
\end{gathered}
$$

and the collective potential function is accordingly computed by $U(\mathbf{x})=\frac{1}{2} \sum_{i \in \vee} \sum_{j \in N_{i}} U_{i j}(\mathbf{x})$.

In (8), $\rho_{h}\left(z /\|R\|_{\sigma}\right)$ is employed to remove the impact of sensors outside of communication range.

In the proposed algorithm, $\omega(z)$ is defined by $\omega(z)= \begin{cases}-p_{0} z^{2}+p_{1} z+p_{2}, & z \leq-t \\ \phi(z), & -t<z<t \\ q_{0} z^{2}+q_{1} z+q_{2}, & z \geq t\end{cases}$

Where $\phi(z)=\frac{1}{2}\left[(a+b) \frac{z+c}{\sqrt{1+(z+c)^{2}}}+(a-b)\right], 0<a \leq b$.

In (11), parameter $\mathrm{c}$ is chosen as $\frac{|a-b|}{\sqrt{4 a b}}$, such that $\omega(0)=\phi(0)=0$. Compared to $\phi(z), \omega(z)$ is emphasized by quadratic functions when $z$ approaches $\pm\|d\|_{\sigma}$. Note that $\omega(z)$ is a piecewise function. To achieve a differentiable $\mathrm{U}(\mathrm{x})$, given $t \geq 0$ and $p_{0}, q_{0} \geq 0$, parameters $p_{i}$ and $q_{i}$ for $\mathrm{i}=1,2$ are chosen in such a way that $\omega(z)$ and its first-order differential are both continuous at $\pm t$. Thereby, we have $\quad p_{1}=\frac{1}{2} \cdot \frac{a+b}{\left[1+(c-t)^{2}\right]^{\frac{3}{2}}}-2 p_{0} t$

$$
\begin{gathered}
q_{1}=\frac{1}{2} \cdot \frac{a+b}{\left[1+(c+t)^{2}\right]^{\frac{3}{2}}}-2 q_{0} t \\
q_{2}=\phi(t)-q_{0} t^{2}-q_{1} t .
\end{gathered}
$$

$$
p_{2}=\phi(-t)+p_{0} t^{2}+p_{1} t
$$

Parameters $p_{0}$ and $q_{0}$ determine, respectively, the strength of repulsive or attractive force applied 
on sensors when the distance between a pair of sensors approaches 0 or goes beyond the desired value $\mathrm{d}$. In practice, we can assume that $p_{0}=q_{0} \geq 0$.Parameter $t$ is chosen within the interval $\left[0,\|d\|_{\sigma}\right]$. When $t$ is closer to 0 , a more stringent constraint is imposed on neighboring sensors, such that their distance gets closer to $\mathrm{d}$, yielding a satisfactory configuration. Using $\mathrm{U}(\mathrm{x})$, stimulus ui of (5) is expressed as

$$
\begin{aligned}
\mathbf{u}_{i} & =-\nabla_{\mathbf{x}_{i}} U(\mathbf{x})+\sum_{j \in \mathbf{N}_{i}} a_{i j}\left(\mathbf{v}_{j}-\mathbf{v}_{i}\right) \\
& -c_{1}\left(\mathbf{x}_{i}-\mathbf{x}_{T}\right)-c_{2}\left(\mathbf{v}_{i}-\mathbf{v}_{T}\right) \\
& =\sum_{j \in \mathbf{N}_{i}} \varphi\left(\left\|\mathbf{x}_{j}-\mathbf{x}_{i}\right\|_{\sigma}\right) \sigma_{\varepsilon}\left(\mathbf{x}_{j}-\mathbf{x}_{i}\right) \\
& +\sum_{j \in \mathbf{N}_{i}} a_{i j}\left(\mathbf{v}_{j}-\mathbf{v}_{i}\right) \\
& -c_{1}\left(\mathbf{x}_{i}-\mathbf{x}_{T}\right)-c_{2}\left(\mathbf{v}_{i}-\mathbf{v}_{T}\right)
\end{aligned}
$$

The first term in (16) is introduced such that neighboring sensors tend to be equally distant from each other. The second term represents a velocity consensus term such that neighboring sensors move along. The third and fourth terms are used to track the movement of the TOI.

\section{Based on aggregation algorithm[11]}

Deterministic annealing technology is the California Institute of Technology, Dr. K Rose In 1990, it was an important branch of the law of nature. For solving a minimization problem:

$$
\min E=E(x)
$$

Where $x$ can be continuous, discrete or mixed; E $(x)$ can be regarded as the energy of a certain system. First construct a free energy function $\mathrm{F}(x, \mathrm{~T})$ when the type of deterministic annealing minimization problem (17) as the minimum energy solution of a physical system state. From the above analysis, at a certain temperature, the change of the system state is always in the direction of the reduction of the free energy, and when the system reaches the equilibrium state, the function can reach the minimum. When $\mathrm{F}(\mathrm{x}, \mathrm{T})$ is a continuous mapping, the global minimal point $x_{\min }(T)$ is $\mathrm{T}$ continuous mapping[11]. When set $T=\infty$, the global $\mathrm{F}(\mathrm{x}, \mathrm{T})$ of the most advantages is extremely easy to find, $F(x, 0)=E(x)$. The deterministic annealing technique, is at each temperature, the system in $T=T+\Delta T$ when free to minimization of state $x_{\min }(T+\Delta T)$ as the starting point, by solving $\min F(x, T)$, very small to simulate the system to achieve the equilibrium process. With the decrease of $T$, the global minimizer is constantly changing, when $T$ changes $\Delta T$ is very small, that $X_{\text {min }}(T)$ at where the local minima in the region, so at temperature $T$, can be used $X_{\text {min }}(T+\Delta T)$ as the initial solution is $\mathrm{F}_{\min }(\mathrm{x}, \mathrm{T})$. When the $\mathrm{T}$ decreases the speed reasonably, it is considered that the global minimum of $\lim _{T \rightarrow 0} X_{\min }(T)$ is the Formula (17).

For the aggregation problem, the free energy function is defined by deterministic annealing 
technique: $F\left(y_{1}, y_{2}, \mathbf{K}, y_{M}, \beta\right)= \begin{cases}\frac{1}{M} \sum_{i=1}^{N} \sum_{j=1}^{M}\left(d\left(x_{i}, y_{j}\right)^{2}\right) & \beta=0 \\ -\frac{1}{\beta} \sum_{i=1}^{N} \ln \sum_{j=1}^{M} e^{-\beta\left(d\left(x_{i}, y_{j}\right)\right)^{2}} & 0<\beta<+\infty \\ \sum_{i=1}^{N} \sum_{j=1}^{M}\left(d\left(x_{i}, y_{j}\right)\right)^{2} & \beta=+\infty\end{cases}$

$X_{i} \in C_{j}, \quad P\left(x_{i} \in C_{j}\right)=\frac{e^{-\beta\left(d\left(x_{i}, y_{i}\right)\right)^{2}}}{\sum_{k=1}^{M} e^{-\beta\left(d\left(x_{i}, y_{k}\right)\right)^{2}}}$

Assume $\quad d\left(x, y_{j}\right)=\left\|x-y_{j}\right\|=\left(\sum_{k=1}^{n}\left(x(k)-y_{j}(k)\right)^{2}\right)^{\frac{1}{2}} \quad, \quad$ When $\quad \beta=0$, $F\left(y_{1}, y_{2}, \mathbf{K}, y_{M}, \boldsymbol{\beta}\right) \quad$ for $\left(y_{1}, y_{2}, \mathbf{K}, y_{M}\right)$ is the continuous differentiable convex function,according to the traditional optimization method, the overall merits of the most easily is obtained. when $\beta \in[0,+\infty), F\left(y_{1}, y_{2}, \mathbf{K}, y_{M}, \beta\right)$ for $\left(y_{1}, y_{2}, \mathbf{K}, y_{M}\right)$ is continuous differentiable, The continuous mapping of the minimal point and minimum of $F\left(y_{1}, y_{2}, \mathbf{K}, y_{M}, \beta\right)$ to $\beta$. At the minimal point of $F\left(y_{1}, y_{2}, \mathbf{K}, y_{M}, \beta\right)$ the first order necessary condition is satisfied: $\frac{\partial F\left(y_{1}, y_{2}, K, y_{M}, \beta\right)}{\partial y_{j}}=0 \quad(\forall j)$

Bring (18) to (20): $\quad y_{j}=\frac{\sum_{i=1}^{N} x_{i} p\left(x_{i} \in C_{j}\right)}{\sum_{i=1}^{N} p\left(x_{i} \in C_{j}\right)}$

$p\left(x_{i} \in C_{j}\right)$ by (19).when $\beta=0, p\left(x_{i} \in C_{j}\right)=\frac{e^{-\beta\left(d\left(x_{i}, y_{j}\right)\right)^{2}}}{\sum_{j^{\prime}=1}^{M} e^{-\beta\left(d\left(x_{i}, y_{j^{\prime}}\right)\right)^{2}}}=\frac{1}{M}$

$y_{j}=\frac{1}{M} \sum_{i=1}^{N} x_{i}$ is the global merit of $F\left(y_{1}, y_{2}, \mathbf{K}, y_{M}, \boldsymbol{\beta}\right)$. 
Formula (21) can get: $y_{j}^{(k+1)}=\frac{\sum_{i=1}^{N} x_{i} p\left(x_{i} \in C_{j}^{(k)}\right)}{\sum_{i=1}^{N} p\left(x_{i} \in C_{j}^{(k)}\right)}$

Formula (23) is the optimal iteration result.According to the traditional optimization method, the overall merits of the most easily is obtained.

This algorithm can improve the robustness of wireless sensor networks, and then extend the life of the network. The problem of node aggregation is studied, and a distributed aggregation algorithm (DADA) based on deterministic annealing is proposed, The algorithm has good adaptability and expansibility.

\section{Monte Carlo box localization algorithm based on differential evolution (DEMCB)[12]}

Based on the difference in the evolution of Monte Carlo localization box algorithm (also known as DEMCB algorithm ) is on the traditional MCB algorithm (MCL algorithm is a simplified and improved) based on. In the sampling stage into the differential evolution algorithm to sample the initiative to the true location of the nodes near, and not be filtered. Because of this no need to repeat the sampling, reduce the power consumption of computing nodes. At the same time. In the MSL algorithm inspired, the ordinary nodes introduced concept of confidence. And ordinary neighbor node of the confidence degree of information to the node sampling sample weights are calculated. In order to improve the ordinary nodes in the low density of anchor nodes positioning performance. Basic steps of DEMCB algorithm of the follows: establish sampling box constraints and obtain the initial sample; ordinary node localization confidence: the combination of neighbor anchor node information and common neighbor nodes confidence degree of computing nodes sample weights were calculated. To sample weight in order to adapt to the function values are performed on the samples and the differential evolution algorithm; sample weighted average as the node localization estimates that the final calculation of the maximum node positioning error.

\section{Establish sampling box constraints}

In order to improve the sampling efficiency of Langendoen.Baggio, a Monte Carlo MCL (MCB) method is proposed[13], which is based on the prediction stage to narrow the sampling area.. If a node has several anchors, the anchor box $x_{\min }, x_{\max }, y_{\min }, y_{\max }$ is as follows:

$$
\begin{aligned}
& x_{\text {min }}=\max _{i=1}^{n}\left\{x_{i}-r\right\}, x_{\text {max }}=\min _{i=1}^{n}\left\{x_{i}+r\right\} \\
& y_{\text {min }}=\max _{i=1}^{n}\left\{y_{i}-r\right\}, y_{\text {max }}=\min _{i=1}^{n}\left\{y_{i}+r\right\}
\end{aligned}
$$

$\left(x_{i}, y_{i}\right)$ is the coordinates of a neighbor anchor node. For the two hop neighbor anchor nodes, the change in the type of $2 \mathrm{r}$ is only needed. 
At the same time, the position estimation $\left(x_{t-1}, y_{t-1}\right)$ and the maximum position $\left(e r r_{x, t-1}, e r r_{y, t-1}\right)$ can also be used to narrow the sampling box, taking $X_{\min }$ as an example:

$$
X_{\min }=\max \left\{x_{\min }, x_{t-1}-v_{\max }-\operatorname{err}_{x, t-1}\right\}
$$

\section{Node confidence}

The confidence of the nodes is used to measure the positioning performance of a common node. Usually, the smaller the sample constraint, the more likely the sample is closer to the node's true location, and the location error is the smaller, the sampling constraint is the larger, and the error is the largre.

This paper choose Diagonal line anchor box and diagonal of the node $\left(0, X_{\text {_ }}\right.$ Range $) \times\left(0, Y_{\text {_ }}\right.$ Range $)$ to deploy the confidence of the node :

$$
\operatorname{credit}(i)=1-\operatorname{diag}_{i} / \operatorname{diag}
$$

Where $\operatorname{diag}_{i}$ is the diagonal length of the anchor box at the moment, and diag is the diagonal length of the node deployment area. At the beginning, the confidence level of the common node is set to 0 . From the formula (26), the reliability of the node is higher when the node's degree of confidence is higher, the localization performance of the node is better.

\section{Calculation result}

After the sampling box gets $\mathrm{N}$ sample, the weight of the sample is calculated according to the neighbor anchor node information received by the node and the information of the ordinary neighbor node.. Suppose the node view is $o_{t}=S \cup T U U S$, where $\mathrm{S}$ is a neighbor anchor node set, T is two hop anchor node set, US is a common neighbor node set.

Thus, the weights of the sample I of T moments are calculated as follows:

$\boldsymbol{\omega}_{t}^{i}=p\left(o_{t} \mid I_{t}^{i}\right)=\prod_{s \in S, T} p\left(o_{t} \mid I_{t}^{i}\right) \sum\left\{\operatorname{credit}(s), s \in U S \mid d\left(s, I_{t}^{i}\right)<r\right\}$

\section{Maximum positioning error}

After the sensor node calculates its position information at the present time, the maximum position error of $\left(e r r_{x, t}, e r r_{y, t}\right)$ can be calculated from the current time constraint box. 


$$
\begin{aligned}
& \operatorname{err}_{x, t}=\max \left\{x_{t}-x_{\text {min }}, x_{\text {max }}-x_{t}\right\} \\
& \operatorname{err_{y,t}}=\max \left\{y_{t}-y_{\text {min }}, y_{\text {max }}-y_{t}\right\}
\end{aligned}
$$

\section{Conclusion}

(II)approach adjusts the relative distance of each sensor to its neighbors to maintain a equilateral triangle configuration, that is proven to be the best hole-free structure with maximal coverage.(III) show that the aggregation formed by algorithm is appropriate scale. The time cost is moderate growth. It is scalable and self-adjustable.(IV) has greatly improved the average localization error and the speed of convergence of the nodes. At the same time, this algorithm can be well applied to the network environment of low anchor node density, which can reduce the cost of sensor network.

\section{Acknowledgment}

This work was supported in part by the National Nature Science Foundation of China under grants 61101158.

\section{References}

[1] C.-Y. Chong and S. Kumar, "Sensor networks: Evolution, opportunities, and challenges," Proc. IEEE, vol. 91, no. 8, pp. 1247-1256, Aug. 2003.

[2] C.-F. Huang and Y.-C. Tseng, "The coverage problem in a wireless sensor network," Mobile Networks and Applications, vol. 10, no. 4, pp. 519-528,2005.

[3] S. Meguerdichian, F. Koushanfar, M. Potkonjak, and M. B. Srivastava, "Coverage problems in wireless ad-hoc sensor networks," Proc. INFOCOM'01,Anchorage, USA, Apr. 2001.

[4] S. S. Dhillon and K. Chakrabarty, "Sensor placement for effective coverage and surveillance in distributed sensor networks," Proc. WCNC'03, New Orleans, USA, Mar. 2003.

[5] T. Clouqueur, V. Phipatanasuphorn, P. Ramanathan and K. K. Saluja, "Sensor deployment strategy for target detection," Proc. WSNA'02, Atlanta, Georgia,Sep. 2002.

[6] Q. Wu, N. S. Rao, X. Du, S. S. Iyengar, and V. K. Vaishnavi, "On efficient deployment of sensors on planar grid," Computer Communications vol. 30, pp. 2721-2734, 2007.

[7] A. Howard, M. J. Matari'c, and G. S. Sukhatme, "An incremental self-deployment algorithm for mobile sensor networks," Autonomous Robots, vol. 13,pp. 113-126, 2002.

[8] G. Wang, G. Cao, and T. La Porta, "Movement-assisted sensor deployment," IEEE Trans. Mob. Comput., vol. 5, pp. 640-652, 2006.

[9] M. Ma and Y. Yang, "Adaptive triangular deployment algorithm for unattended mobile sensor networks,” IEEE Trans. Comput., vol. 56, pp. 946-958,2007.

[10] R. Olfati-Saber, "Flocking for multi-agent dynamic systems: algorithms and theory," IEEE Trans. Automat. Contr., vol. 51, pp. 401-420, 2006.

[11] huai-hu Cao, yan-mei Zhang, “Aggregation Algorithm for Mobile Sensor Network Based on Deterministic Annealing,” in chinese .Computer engineering,vol.38,pp.88-90,2012.

[12] wang-dong MAO, wen-zhen YUE, neng-hai YU,“A new node localization algorithm for mobile wireless sensor networks," in chinese.Data Communications, vol 2,pp15-19,2014

[13] Baggio Aline, Langendoen Koen. Monte Carlo localization for mobile wireless sensor networks[J]. Ad boc Networks, vol.6,pp.718-732,2008 
